\title{
20nm Silicon nanorods fabricated by reactive ion etch
}

\author{
Eih-Zhe Liang, Chao-Jei Huang, and Ching-Fuh Lin* \\ Graduate Institute of Electro-optical Engineering, \\ * Also with Department of Electrical Engineering and Graduate Institute of Electronics Engineering \\ National Taiwan University, Taipei, Taiwan, Republic of China
}

\begin{abstract}
Silicon nanorods with 20nm diameter size is fabricated on single-crystalline silicon surface by reactive ion etch. Silicon dioxide nanoparticle is dispersed on surface by spin-coating to form monolayer and is used as the etching mask. $\mathrm{SF}_{6}$ and $\mathrm{O}_{2}$ are used for reactive ion etching. The gas mixture ratio is optimized for anisotropic etching of nano-structures. Surface damage of the crystal lattice is monitored using microwave-reflectance photo-conductance decay technique with the removal and probe scheme. Vertical etch damage region is measured to be within $30 \mathrm{~nm}$.

Index Terms - Nanotechnology, plasma material-processing applications, silicon, surface contamination.
\end{abstract}

\section{INTRODUCTION}

Nanostructures with decananometer scale have been demonstrated to have optical gain [1] and to be a key element in single electron transistor [2]. Silicon nanorods can be prepared by dc-sputtering [3], hot-wired CVD [4], e-beam lithography, and near-field optical lithography [5]. We demonstrate the fast fabrication of silicon nanorods with $20 \mathrm{~nm}$ size on single crystalline silicon by reactive ion etch. Silicon dioxide nanoparticle is used as the etch mask. With this method, large area and high density silicon nanorods can be easily fabricated.

For future electronic devices, it is a trend to shrink device size into decananometer scale. However, large surface to volume ratio emerges and surface damage becomes very influential to device performance. It is also appealing to investigate the applicability of dry etch in the nanostructures, including surface damage and dimensional control. Etch techniques such as reactive ion etch (RIE) must both optimize anisotropy and minimize damage of underlying material. Here the crystal damage by ion bombardment is monitored by microwave-reflection photoconductance decay with a removal and probe scheme.

\section{MASK PREPARATION}

In the first place, a etch mask with decananometer size is prepared. Silicon dioxide nanoparticle is used to mask dry etch of silicon. The $\mathrm{SiO}_{2}$ particles have a sphere shape with $20 \sim 30 \mathrm{~nm}$ diameter and dispersed in isopropanol with $1 \%$ in weight. Silicon wafer with one side polished is treated with buffered oxide etch $(\mathrm{BOE}, \mathrm{HF}: \mathrm{NHF}=1: 6)$ to remove native oxide and preserve a hydrophobic surface. The nanoparticle solution is heated above $50^{\circ} \mathrm{C}$ and applies to silicon surface by spin-coating.

To be noted, raising temperature of solution is crucial in obtaining a uniform monolayer of silicon dioxide nanoparticle. It is attributed to the reduction of mutual attraction of nanoparticles by enhancement of Brownian motion in solution.

As shown in Fig. 1a), relative magnitude of attraction force between adjacent nanoparticles $F_{o x}$ and attraction between nanoparticle and silicon surface $F_{s i}$ will lead to different morphology. When $F_{s i}>F_{o x}$ in Fig $1 b$ ), the spin-coated layer is monolayer. It is measured to have $25 \mathrm{~nm}$ thickness with effective refractive index 1.46 at $633 \mathrm{~nm}$ by ellisometer. When $\mathrm{F}_{\mathrm{si}}<\mathrm{F}_{\mathrm{ox}}$ in Fig 1c), the spin-coated layer cannot form long range film within $100 \mathrm{~nm}$ area.

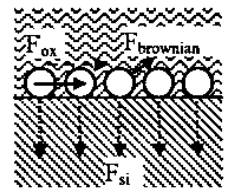

a)

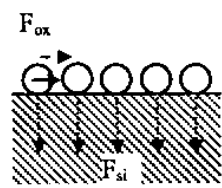

b) $\mathrm{F}_{\mathrm{si}}>\mathrm{F}_{\mathrm{ox}}$

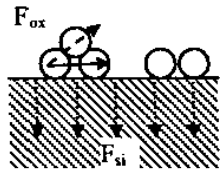

c) $\mathrm{F}_{\mathrm{si}}<\mathrm{F}_{\text {ox }}$
Fig. 1. a) Forces in nanoparticle solution applied to silicon surface $b, c)$ in spin-coated layer.

The criterion of monolayer formation depends on temperature, solvent, concentration of solution. It is empirical that choosing solvent with higher viscosity, raising temperature and adequate concentration will lead to uniform monolayer within an area up to $5 \times 5 \mathrm{~cm}^{2}$. Only solvents with viscosity coefficient greater than isopropanol can produce monolayer if the solution is heated below the boiling point. 


\section{DRY ETCH}

After etch mask is prepared, the wafer is placed in RIE chamber and dry etch is performed. Etching parameters like gas mixture ratio, RF power, and gas ambient pressure are optimized for anisotropy. Anisotropy is defined as $1-V_{L} / V_{H}$, where $V_{L}$ is the laterally etched width and $V_{H}$ is the vertically etched depth. $\mathrm{SF}_{6}$ and $\mathrm{O}_{2}$ are used. Relation of gas mixture ratio and anisotropy is shown in Fig. 2. With lower $\mathrm{SF}_{6}$ percentage, anisotropy is better, up to $90 \%$. It is worth noting that dry etch of silicon using $\mathrm{SF}_{6}$ and $\mathrm{O}_{2}$ can have anisotropy without undercut beneath the mask if the etch depth is less than $200 \mathrm{~nm}$. The edge profile is slightly trapezoidal.

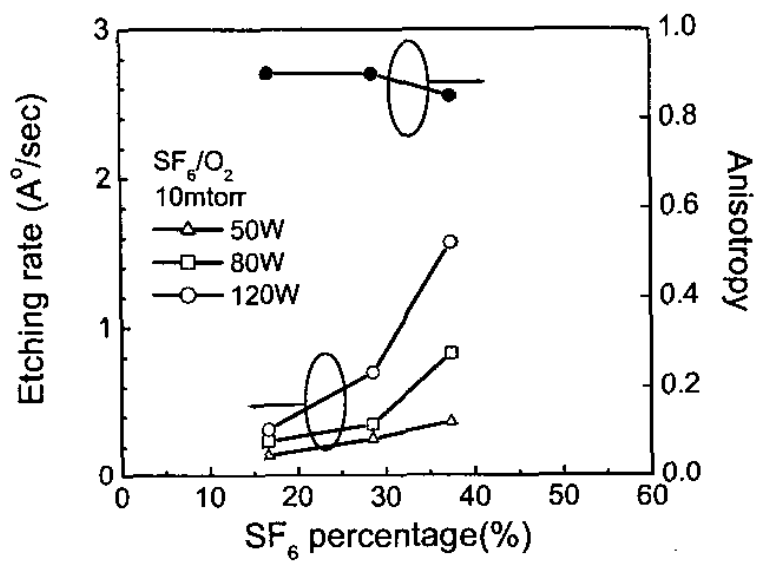

Fig. 2. Etching rate and anisotropy vs. gas mixture ratio.

SEM photo (top view) of as-spun silicon dioxide nanoparticle mask is shown in Fig. 3a). The lateral arrangement is random due to large size dispersion of $40 \%$. However the nanoparticles do form a monolayer without clustering. The packing density is counted to be $10^{11} \mathrm{~cm}^{-2}$.

The etched wafer is treated with BOE to remove mask and shown in Fig. 3b) and Fig. 3c). The silicon nanorods has diameter $15 \sim 25 \mathrm{~nm}$ and height $20 \mathrm{~nm}$. STM image with current feedback mode in Fig. $3 \mathrm{~d}$ ) is used to investigate steepness of etched profile. The nanorods have trapezoidal shape and the top is slightly rounded due to pattern transfer of spherical mask.

The mean spacing of adjacent nanorods is about $30 \mathrm{~nm}$. It is a copy of adjacent nanoparticles and is influenced only by solution concentration. When concentration is larger than $1 \%$ in weight, nanoparticles tend to pack onto each other and form double layer. When concentration is smaller, spacing of adjacent nanorods becomes longer. It cannot sustain uniform thin film with large area coverage if concentration is lower than $0.5 \%$ in weight.

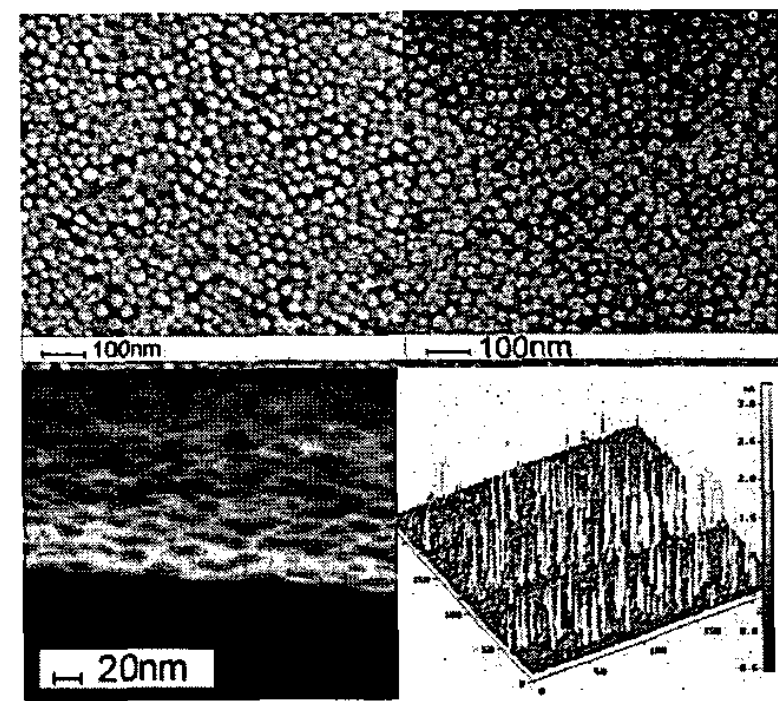

Fig. 3. a) SEM image of silicon dioxide nanoparticles spin-coated film b) silicon nanorods with average diameter size $20 \mathrm{~nm}$.(top view) c) (side view) d) STM image with current feedback mode.

\section{ETCH DAMAGE}

It is important to minimize etch damage during fabrication of decananometer structure. Surface to volume ratio is inversely proportional to structure dimension, and becomes ten times larger than in hundred nanometer scale. This means surface states have ten times greater influence.

It is known that dry etch techniques have polymeric surface contamination and lattice damage. In reactive ion etch case, surface is modified into complex bonding, for example $\mathrm{Si}-\mathrm{F}$ and $\mathrm{Si}-\mathrm{O}$ bonding in $\mathrm{SF}_{6}$ and $\mathrm{O}_{2}$ gas ambient. This contamination layer is estimated to be as shallow as $5 \sim 8 \mathrm{~nm}$ [6].Lattice damage is deeper since reactive ion penetrates into inner crystal volume. The penetration depth depends on kinds of ion and external RF power.

Microwave-reflectance photoconductance decay (MWPCD) is used to monitor the etch damage. The surface quality, i.e. surface traps or surface recombination velocity, can be derived from measured minority carrier life time. By a removal and probe scheme, defects of inner crystal volume can be measured.

In the investigation, a one-side polished float zone silicon wafer with doping $10^{-15} \mathrm{~cm}^{-3}$ and orientation (100) is used. Its carrier lifetime is measured by MWPCD after its immersion in $8 \%$ molar iodine/methanol [7]. The microwave probe is at $10 \mathrm{GHz}$ and excitation source is $532 \mathrm{~nm}$ second harmonic generation of $\mathrm{Nd}: \mathrm{YAG}$ pulse laser. Bulk lifetime can be estimated by changing peak excitation intensity up to $400 \mathrm{~kW} / \mathrm{cm}^{2}$. 
The wafer has the thickness of $525 \mu \mathrm{m}$ and the carrier lifetime of the bulk crystal is $1.3 \mathrm{~ms}$. It is treated by aforementioned $\mathrm{SF}_{6} / \mathrm{O}_{2}$ dry etch at pressure $10 \mathrm{mtorr}$ and RF power $120 \mathrm{~W}$ and the etching depth is $20 \mathrm{~nm}$. Damaged silicon is removed by $\mathrm{KOH}(45 \%$ in weight) wet etch at $35^{\circ} \mathrm{C}$, with etching rate $2.1 \mathrm{~nm} / \mathrm{sec}$ [8]. Alkaline solution will leave surface hydrophilic and increase passivation effect of iodine/methanol. After the removal of the presumed damage depth is reached, the wafer is rinsed by de-ionized water and placed into iodine/methanol. Its carrier lifetime is measured and afterwards placed back to wet etch bath.

Measured lifetime $\tau_{\mathrm{PCD}}$ can be expressed by

$$
\frac{1}{\tau_{P C D}}=\frac{1}{\tau_{B U L K}}+\frac{S_{1}+S_{2}}{W},
$$

where $\tau_{\mathrm{BULK}}$ is the bulk lifetime, $\mathrm{W}$ is the wafer thickness, $S_{1}$ and $S_{2}$ are surface recombination velocity of damaged front side and undamaged backside. Please note that $S_{2}$ is very small $<50 \mathrm{~cm} / \mathrm{s}$ as determined before dry etch. $S_{1}$ is then calculated from $\tau_{\mathrm{PCD}}, \tau_{\mathrm{BULK}}$ and $\mathrm{W}$.

Derived surface recombination velocity is depicted versus removed thickness. It can be shown that highly damaged silicon is about $30 \sim 40 \mathrm{~nm}$. This can be compared with previous reported $16 \sim 36 \mathrm{~nm}$ damage thickness [6]. However, our sensitive measurement suggests that the lightly damaged part extends into more than $100 \mathrm{~nm}$.

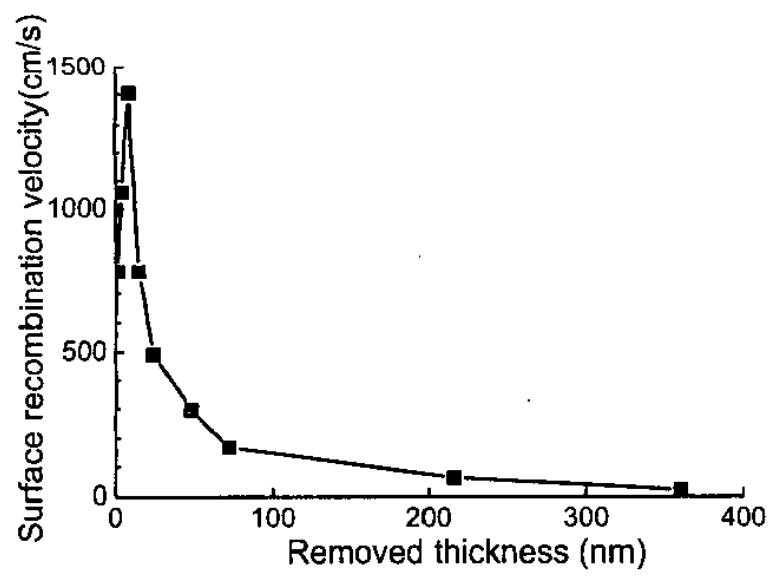

Fig. 4. Derived surface recombination velocity of damaged front side versus different removed thickness by $\mathrm{KOH}$ wet etch.

Although vertical etch damage can be determined by the above mentioned method, lateral damage can only be estimated. Considering $90 \%$ anisotropy of our $\mathrm{Sf}_{6} / \mathrm{O}_{2}$ recipe, ten percent of highly damage thickness is estimated to be $3 \mathrm{~nm}$. In such case, inner part of quantum dots may preserve its original float-zone quality.

\section{CONCLUSION}

We demonstrate silicon nanorods with $20 \mathrm{~nm}$ diameter size on single-crystalline silicon surface by reactive ion etch. Silicon dioxide nanoparticle is spin-coated onto silicon surface and proven to form monolayer. This monolayer is used as etching mask. Silicon etch using $\mathrm{SF}_{6}$ and $\mathrm{O}_{2}$ gas mixture ratio can have $90 \%$ anisotropy. Dry etch damage of underlying silicon is monitored by microwave-reflectance photo-conductance decay technique. At vertical part, there is $30 \mathrm{~nm}$ highly damaged silicon and $100 \mathrm{~nm}$ lightly damaged silicon.

\section{ACKNOWLEDGEMENT}

The authors wish to acknowledge support of National Science Council under contract number NSC 92-2622-L-002-001.

\section{REFERENCES}

[1] L.D Negro, L. Pavesi, G. Pucker, G. Franzo, F. Priolo, "Optical gain in silicon nanocrystals" Optical Materials, vol. 17, pp. 41-44, 2002.

[2] A. Dutta, S. Oda, Y. Fu, M. Willander "Electron transport in nanocrystalline $\mathrm{Si}$ based single electron transistors" Jpn. J. Appl. Phys. vol. 39, 4647-4650, 2000.

[3] Y. Zhu, G.S. Cheng, L.D. Zhang "Preparation and formation mechanism of silicon nanorods" J. Mater. Sci. Lett. vol. 17, 1897-1898, 1998.

[4] T.C. Wong, J.J. Wu "Low temperature syntheses of nano-crystalline silicon film and $\mathrm{Si}$ nanorods by hot-wire CVD" Materials Research Society Symposium Proceedings, vol. 715, 179-184, 2002.

[5] Y. Yin, B. Gates, Y. Xia "A soft lithographic approach to the fabrication of single crystalline silicon nanostructures with well-defined dimensions and shapes" Materials Research Society Symposium - Proceedings, vol. 636, D421-D426, 2001.

[6] K.A. Reihardt, S.M. Kelso "The use of beam profile reflectometry to determine depth of silicon etch damage and contamination" Proceedings of SPIE, vol. 2638, 147-158, 1995.

[7] A.W. Stephen, M.A. Green "Effectiveness of 0.08 molar iodine in ethanol as means of chemical surface passivation for photoconductance decay measurements" Solar Energy Materials and Solar Cells, vol. 45, pp.255-265, 1997.

[8] L.D. Dyer, G.J. Grant, C.M. Tiption, A.E. Stephens, "A comparison of silicon wafer etching by $\mathrm{KOH}$ and acid solutions" J. Electrochem. Soc., vol. 136, 3016-3018, 1989. 